\title{
Probing nanoscale ion dynamics in ultrathin films of polymerized ionic liquids by broadband dielectric spectroscopy
}

\author{
Maximilian Heres, Tyler Cosby, Emmanuel Mapesa, Joshua Sangoro*
}

\section{Thin film quality analysis}

Film thickness and quality of the polymerized 1-vinyl-3-ethylimidazolium bis(trifluoromethylsulfonyl)imide ionic liquid thin films were investigated using a Nanoscope Multimode atomic force microscope (AFM). The described broadband dielectric spectroscopy (BDS) measurements using nano structured electrodes are extremely sensitive to dust, which prohibits the film sample to be handled outside of a clean bench for a prolonged period of time, prior to BDS measurements. AFM measurements were therefore conducted after the BDS measurements. Figure S1 shows the AFM measurement of a depth profile created by scratching the film surface with a sterile scalpel blade, in order to determine film thickness. Dust particles are visible as white spots on the film.
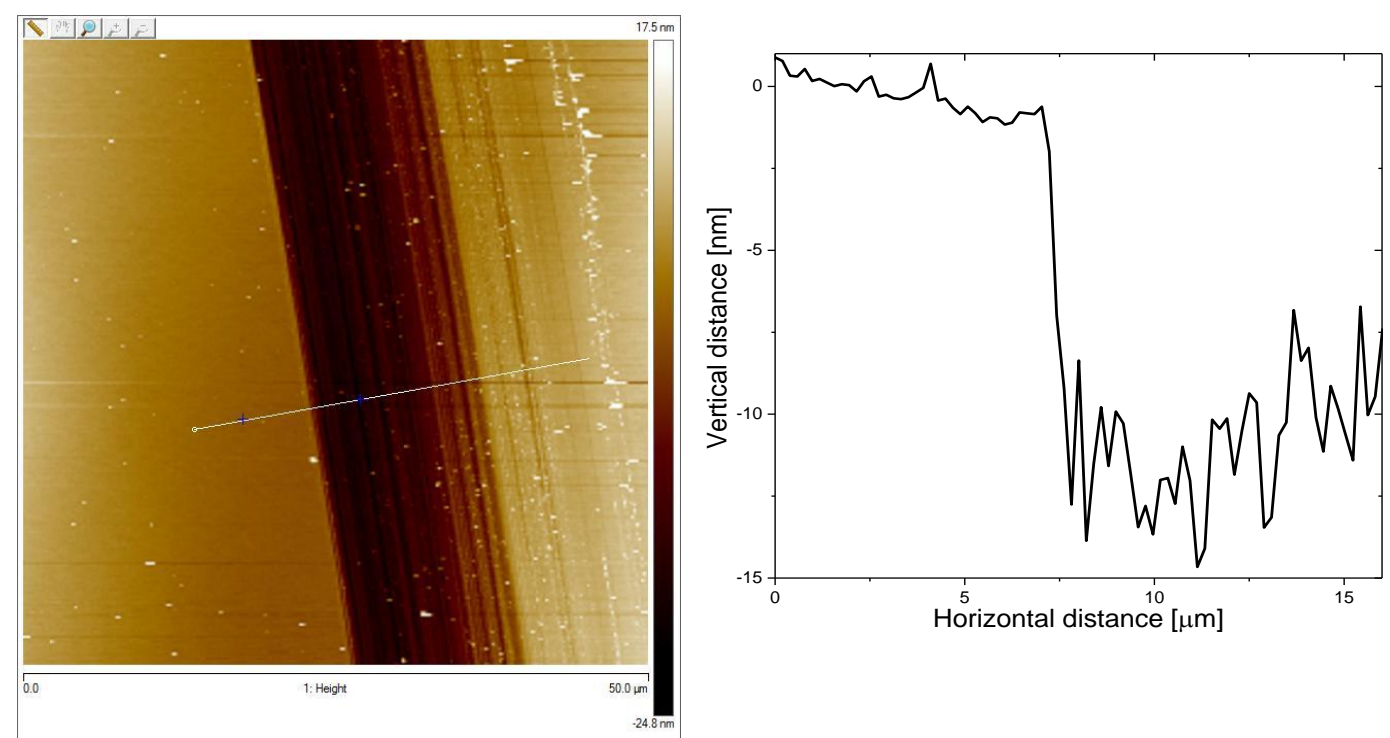

Figure S1: Depth profile of a $12 \mathrm{~nm}$ thick film of polymerized 1-vinyl-3ethylimidazolium bis(trifluoromethylsulfonyl)imide ionic liquid measured over a $50 \mu \mathrm{m} \times 50 \mu \mathrm{m}$ area. The depth profile indicated by a white line on the AFM image (left) is shown as a function of distance (right).

A closer inspection of a $100 \mathrm{~nm} \times 100 \mathrm{~nm}$ area of the same film on a region not affected by the scratch reveals uniform film surface and no de-wetting behavior as 
shown in Figure S2. This film is representative of all film samples measured in this study.

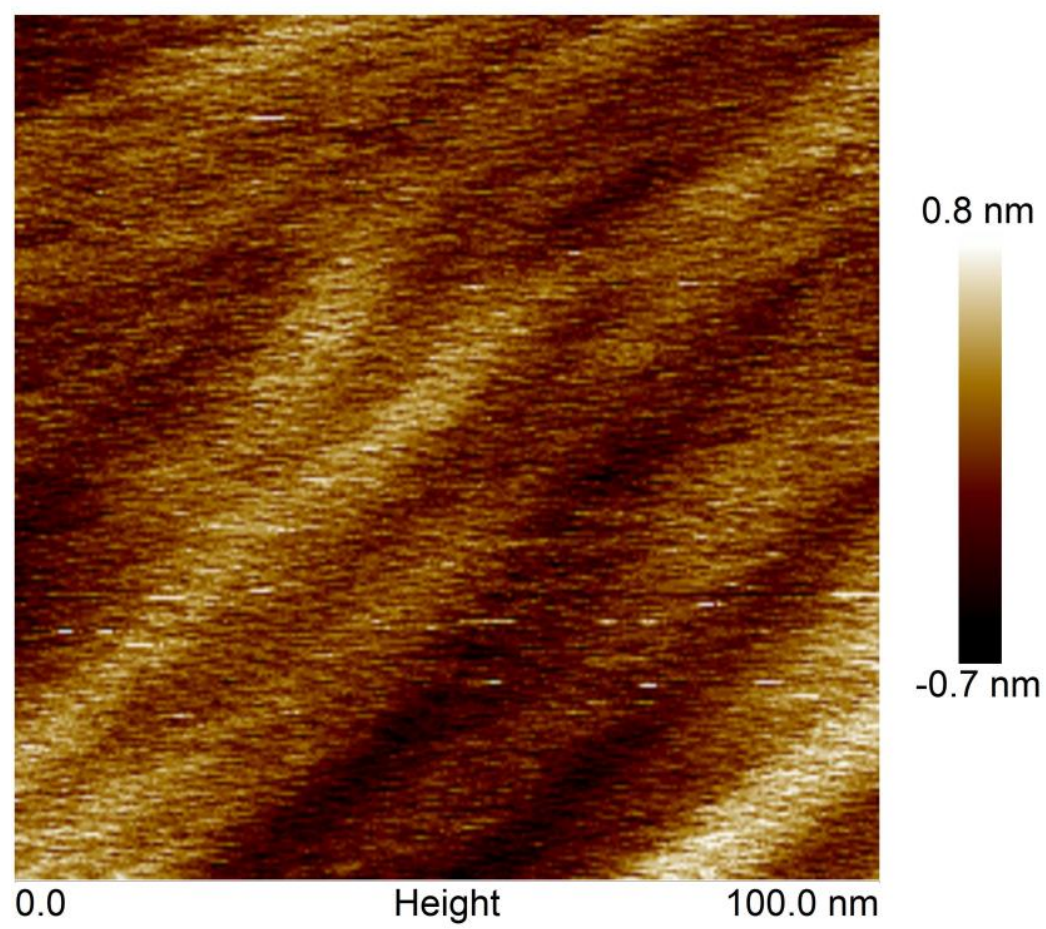

Figure S2: Section of polyIL thin film spanning 100nm x 100nm to reveal no dewetting of the polymer film after annealing and measurement by broadband dielectric spectroscopy. 